\title{
MCC950, the Selective Inhibitor of Nucleotide Oligomerization Domain-Like Receptor Protein-3 Inflammasome, Protects Mice against Traumatic Brain Injury
}

\author{
Saifudeen Ismael, Sanaz Nasoohi, and Tauheed Ishrat ${ }^{1,3}$
}

\begin{abstract}
Nucleotide oligomerization domain (NOD)-like receptor protein-3 (NLRP3) inflammasome may intimately contribute to sustaining damage after traumatic brain injury (TBI). This study aims to examine whether specific modulation of NLPR3 inflammasome by MCC950, a novel selective NLRP3 inhibitor, confers protection after experimental TBI. Unilateral cortical impact injury was induced in young adult C57BL/6 mice. MCC950 $(50 \mathrm{mg} / \mathrm{kg}$, intraperitoneally) or saline was administration at 1 and $3 \mathrm{~h}$ post-TBI. Animals were tested for neurological function and then sacrificed at 24 or $72 \mathrm{~h}$ post-TBI. Immunoblotting and histological analysis were performed to identify markers of NLRP3 inflammasome and proapoptotic activity in pericontusional areas of the brains at 24 or $72 \mathrm{~h}$ post-TBI. MCC950 treatment provided a significant improvement in neurological function and reduced cerebral edema in TBI animals. TBI upregulated NLRP3, apoptosis-associated speck-like adapter protein (ASC), cleaved caspase-1, and interlukein-1 $\beta$ (IL-1 $\beta$ ) in the perilesional area. MCC950 efficiently repressed caspase-1 and IL-1 $\beta$ with a transient effect on ASC and NLRP3 post-TBI. MCC950 treatment also provided protection against proapoptotic activation of poly (ADP-ribose) polymerase and caspase-3 associated with TBI. A concurrent inhibition of inflammasome priming was also detectable at the nuclear factor kappa B/p65 and caspase-1 level. Our findings support the implication of NLRP3 inflammasome in the pathogenesis of TBI and further suggests the therapeutic potential of MCC950.
\end{abstract}

Keywords: edema; inflammation; MCC950; neurological deficits; NLRP3-inflammasome inhibitor; traumatic brain injury

\section{Introduction}

$\mathbf{T}$ RAUMATIC BRAIN INJURY (TBI) is a major cause of morbidity and mortality in the young population in the United States, contributing to around $35-40 \%$ of all injury deaths, with falls and traffic crashes as the most common external causes. ${ }^{1,2}$ TBI therapies are utterly limited, given that many promising compounds like progesterone analogues have failed in clinical trials because of intolerable side effects and therapeutic insufficiency. ${ }^{3,4}$ Current available therapies in TBI mainly focus on management of secondary complications because the primary necrotic insult is highly irreversible. As such, there is essential requirement to target the deleterious signals leading to programmed cell death and inflammation to rescue the imperiled CNS cells. ${ }^{5-7}$

Numerous concrete evidences suggest that inflammatory responses are likely the prominent and early emerging pathological feature of TBI ${ }^{5,8,9}$ Besides regional inflammatory responses in microglial and neural cells, the insult triggers an invasion of macrophages and neutrophils into the impact area, escalating the tissue damage, at least partly, through proinflammatory cytokines. Over- production of interlukin-1 $\beta$ (IL-1 $\beta$ ) is well documented, providing clear evidence for a pivotal role of this cytokine in TBI-associated inflammation. $^{9-11}$

IL-1 $\beta$ maturation and secretion to systemic circulation is predominantly regulated by a multiprotein complex, the receptor nucleotide oligomerization domain (NOD)-like receptor protein (NLRP3) inflammasome, as a recently described mechanism to govern inflammatory responses during tissue injury. ${ }^{12,13}$ Mainly settled in immune cells, the NLRP3 inflammasome is composed of oligomers of NLRP3, apoptosis-associated speck-like (ASC) adapter protein and pro-caspase-1, to serve as a platform for caspase-1 activation and IL-1 $\beta$ maturation. The NLRP3 inflammasome assembly occurs following stimulation by either pathogen-associated molecular patterns or danger-associated molecular patterns. ${ }^{14}$ According to recent reports, TBI appears to coincide with ASC and NLRP3-inflammasome oligomerization inducing caspase-1 activation as well as IL-1 $\beta$ and IL-18 maturation. ${ }^{15,16}$ Such an implication might approach high significance when NLRP3 has been proposed as a biomarker in cerebrospinal fluid in TBI patients with poor prognosis. ${ }^{17}$ Despite all these emerging backgrounds, little

\footnotetext{
${ }^{1}$ Department of Anatomy and Neurobiology, College of Medicine, The University of Tennessee Health Science Center, Memphis, Tennessee.

${ }^{2}$ Neuroscience Research Center, Shahid Beheshti University of Medical Sciences, Tehran, Iran.

${ }^{3}$ Neuroscience Institute, The University of Tennessee Health Science Center, Memphis, Tennessee.
} 
has been examined in application of NLRP3 inhibitors in TBI. Applying general neuroprotectives like omega-3 fatty acids or telmisartan in experimental TBI, there are some earlier studies to suggest the association with NLRP3 inhibition may account for the therapeutic effects. ${ }^{16,18,19}$ Nevertheless, generally acting as antioxidants or peleiotropic agents, the implication of a wide array of effectors may not be ruled out for such multipotential compounds. ${ }^{20,21}$

Based on such a background, we used the newly developed NLRP3 inhibitor, MCC950, as a highly selective and potent inhibitor, to specify the involvement of NLRP3. ${ }^{22}$ Experimental studies have underlined the beneficial role of MCC950 treatment in several inflammatory disease models. ${ }^{22-24}$ The present study aims to specify whether selective NLRP3-inflammasome inhibition by MCC950 administration confers neuroprotection in experimental TBI. For the existing data addressing NLRP3 activation as early as $24 \mathrm{~h}$ after control cortical impact (CCI) model of experimental TBI, our experiments focus on the acute phase of TBI injury as a window for high therapeutic potentials. Our findings support the contribution of NLRP3 inflammasome in TBI and provide evidences for specific NLRP3 inhibition as a promising therapeutic tool for TBI.

\section{Methods}

\section{Animals and experimental groups}

Wild-type C57B1/6 mice obtained from Jackson Laboratory (Bar Harbor, ME) were maintained in the animal facility in standard conditions at a 12-h light/dark cycle and food and water ad libitum. All animal care and manipulations were carried out according to the Institutional Animal Care and Use Committee. Young adult animals (9-10 weeks) were quarantined and individually housed in cages for 1 week before being randomly assigned to experimental groups: sham, TBI, and TBI + MCC950. Each group was divided into two subgroups for acute and subacute assessment of neurological damage at 24 and $72 \mathrm{~h}$ post-TBI. After the neurological assessments all animals were euthanized at 24 or $72 \mathrm{~h}$ post-TBI, and the brain samples were harvested according to the prerequisite procedure for immunoblotting or histological evaluations. MCC950 (Adipogen Life Sciences, San Diego, CA) was dissolved in sterile saline and dosed in animals ( $50 \mathrm{mg} / \mathrm{kg}$, intraperitoneally) at 1 and $3 \mathrm{~h}$ post-TBI, based on published reports. ${ }^{22,24}$

\section{Induction of traumatic brain injury}

$\mathrm{C} 57 \mathrm{~B} 1 / 6$ mice were subjected to $\mathrm{CCI}$ or the sham surgery under isoflurane (2-3\%) anesthesia. Briefly, mice were placed in a stereotaxic frame (Leica Biosystems, Inc., Buffalo Grove, IL), and a $3.5-\mathrm{mm}$ craniotomy was made in the right parietal bone midway between bregma and lambda with the medial edge $12 \mathrm{~mm}$ lateral to the midline, leaving the dura intact. Mice were impacted at $4 \mathrm{~m} / \mathrm{s}$ with a 20-ms dwell time and 2-mm depression using a 3-mmdiameter convex tip, mimicking a moderate TBI. Sham mice underwent the identical surgical procedures, except the impaction. Body temperature was maintained at $37^{\circ} \mathrm{C}$ using a small animal temperature controller throughout all procedures. The incision was closed with VetBond tissue adhesive (1469SB; 3M Science, Maplewood, MN), and animals were allowed to recover.

\section{Assessment of neurological severity score}

Neurobehavioral assessment was performed at 24 and $72 \mathrm{~h}$ postTBI in a blinded fashion using a 10-point neurological severity score (NSS). ${ }^{18}$ One point was given for each failure to account for the cumulative score with a maximum of 10 . The assessment consisted of 10 different tasks: 1) presence of mono- or hemiparesis; 2) inability to walk on a 3-cm-wide beam; 3) inability to walk on a 2-cm-wide beam; 4) inability to walk on a 1 -cm-wide beam; 5) inability to balance on a 1-cm-wide beam; 6) inability to balance on a round stick $(0.5 \mathrm{~cm}$ diameter $) ; 7)$ failure to exit a $30-\mathrm{cm}$-diameter circle (for $2 \mathrm{~min}$ ); 8) inability to walk a straight line; 9) loss of startle behavior; and 10) loss of seeking behavior.

\section{Assessment of cerebral edema}

Brain water content (BWC), a sensitive measure of cerebral edema, was quantified using the wet-dry method, as detailed by our group. ${ }^{25}$ BWC was estimated in 3-mm coronal sections of the ipsilateral cortex (or corresponding contralateral cortex), centered upon the impact site. Tissue was immediately weighed (wet weight), then dehydrated at $65^{\circ} \mathrm{C}$. The sample was reweighed $48 \mathrm{~h}$ later to obtain a dry weight. Percentage of tissue water content was calculated using the following formula: $\mathrm{BWC}=[$ (wet weight-dried weight)/wet weight] $\times 100 \%$.

\section{Western blotting}

The pericontusional cerebral cortex was microdissected from brains harvested from euthanized animals and immediately frozen in liquid nitrogen. Tissue samples were homogenized in radioimmunoprecipitation assay buffer containing protease inhibitor cocktail and processed for western blotting as previously described. ${ }^{25}$ Thirty micrograms of sample proteins were loaded and separated trough electrophoresis on a 4-20\% polyacrylamide gel (Bio-Rad Laboratories, Hercules, CA). Proteins were then transferred to polyvinylidene difluoride membrane. Membranes were blocked for nonspecific binding and probed with primary antibodies against NLRP3, caspase-1, ASC (1:1000; AG-20B0014; AG-20B-0042; AG-25B-0006; Adipogen Life Sciences), IL-1 $\beta$, caspase-3, phosphorylated protein kinase B (p-Akt), protein kinase B (Akt; 1:000; CST-12242; 9664; 9271; 9272; Cell Signaling Technology, Danvers, MA), poly (ADP-ribose) polymerase (PARP; 1:1000; ab32138; Abcam, Cambridge, MA), nuclear factor kappa B (NF- $\kappa$ B)/p65 (1:1000; Sc-8008; Santa Cruz Biotechnology, Santa Cruz, CA; after Tris-buffered saline with Tween 20 washes, membranes were incubated with horseradishperoxidase-conjugated secondary antibody (1:10,000; SigmaAldrich, St. Louis, MO). The bands were then visualized by means of a chemiluminescent substrate system (Thermo Fisher Scientific, Waltham, MA). The bands' densities were quantified using ImageJ software (NIH, Bethesda, MD) and normalized with the expression of beta actin as the housekeeping gene, and expressed as fold change. (Please see Supplementary Figures 2-7 for full western blots with original markings; online supplementary material may be found at at http://www.liebertpub.com/neu)

\section{Immunofluorescence staining}

Mice were anesthetized with ketamine/xylazine and transcardially perfused with phosphate-buffered saline (PBS) followed by $10 \%$ formalin (Thermo Fischer Scientific). Brains were removed and postfixed in the same fixative overnight at $4^{\circ} \mathrm{C}$ and then with $30 \%$ sucrose in PBS for $72 \mathrm{~h}$. Sunk brains were frozen-sectioned in the coronal plane at a thickness of $10 \mu \mathrm{m}$. Sections were blocked with SerumFree Protein Block (X0909; DAKO, Carpinteria, CA) and then incubated with primary antibodies against NLRP3, caspase-1 (1:200; 1: 250; Adipogen Life Sciences), and IL-1 $\beta$ (1:100; Cell Signaling Technology) overnight at $4^{\circ} \mathrm{C}$ in a humid chamber. After washing, slides were incubated with fluorescent anti mouse secondary antibodies (1:200; 072-04-18-03; Dylight-549; SeraCare KPL Products, Milford, MA) for $1 \mathrm{~h}$ at room temperature and mounted with ProLong ${ }^{\mathrm{TM}}$ Diamond Antifade Mountant with DAPI (Invirogen, Carlsbad, CA), and viewed using a Zeiss 710 confocal laser scanning microscope. Negative controls were prepared by omitting the primary antibodies. Immunofluorescence intensities were quantified using ImageJ software (NIH) in five 
different fields per section digitized from the perilesional area using a $\times 40$ objective lens.

\section{Statistical analysis}

Sample-size estimation. Sample-size determinations were made at alpha $=0.05$, and data are reported as mean \pm standard error. Based on IL- $\beta$ expression value of $4.92 \pm 10 \%$ for saline TBI, and a reduction to $1.87 \pm 10 \%$ for mice treated with MCC950+ TBI, a sample size of 5/group was provided at least $90 \%$ power to detect this difference. One-way analysis of variance was performed to detect any difference in a variable in more than two groups. Tukey's post-hoc was then used to specify the significant differences. A two-tailed Student's $t$-test was applied in cases the variable was compared in two groups like NSS comparison. Statistical significance was accepted at the $95 \%$ confidence level $(p<0.05)$.

\section{Results}

\section{Effect of MCC950 on neurological severity score} and brain water content after traumatic brain injury

NSSs data were compared to track the effect of MCC950 on functional outcome at 24 and $72 \mathrm{~h}$ post-TBI (Fig. 1A). To ensure the normal neurological background, all included animals were tested for the 10-point NSS task before TBI induction and scored with zero. MCC950 treatment showed a trend toward the improvement in the NSS, but significant $(p<0.05)$ improvement was found only at $72 \mathrm{~h}$ compared to the saline-treated group.

Further, MCC950 affects TBI-induced cerebral edema induced by TBI as assessed by BWC. As shown in Figure 1B, TBI mice treated with MCC950 showed a trend to decrease in BWC $(78.04 \pm 2.03)$ compared to saline-treated $(80.98 \pm 0.64)$ counterparts at $24 \mathrm{~h}$ post-TBI; however, the difference did not achieve statistical significance.

\section{MCC950 attenuates inflammasome activation (nucleotide oligomerization domain [NOD]-like receptor protein-3, cleaved caspase-1, apoptosis-associated speck-like adapter protein, and interlukein-1 $\beta$ ) after traumatic brain injury}

According to the illustrated data in Figure 2A-E, our immunoblot analysis showed that expression of the principal constituents of NLRP3 inflammasome, including ASC and NLRP3, as well as their presumptive downstream molecules, cleaved caspase- 1 and IL- $1 \beta$, demonstrate significant overexpression in samples from the pericontusional area after 24 and $72 \mathrm{~h}$ of TBI. Such augmentations were significantly reversed at $24 \mathrm{~h}$ post-TBI in MCC950-treated brains.

To confirm localized NLRP3 inflammasome activation, TBIaffected tissues were probed to detect positive cells for NLRP3, caspase-1, and IL- $\beta$. As shown in Figure 3 representative sections and the following quantification of the fluorescent signal, the obvious augmentations in cytosolic caspase 1 and IL- $1 \beta$ were remarkably reduced in corresponding samples from MCC950 animals either 24 or $72 \mathrm{~h}$ post-injury.

\section{The inhibitory effect of MCC950 coincides} with minute changes in thioredoxin interacting protein and phosphorylated protein kinase $B$ expression after traumatic brain injury

Along our main investigations on MCC950 effects on TBI, we tested the probable involvement of thioredoxin interacting protein (TXNIP) as a pivotal regulator of NLRP3 (Fig. 4). For the existing
A
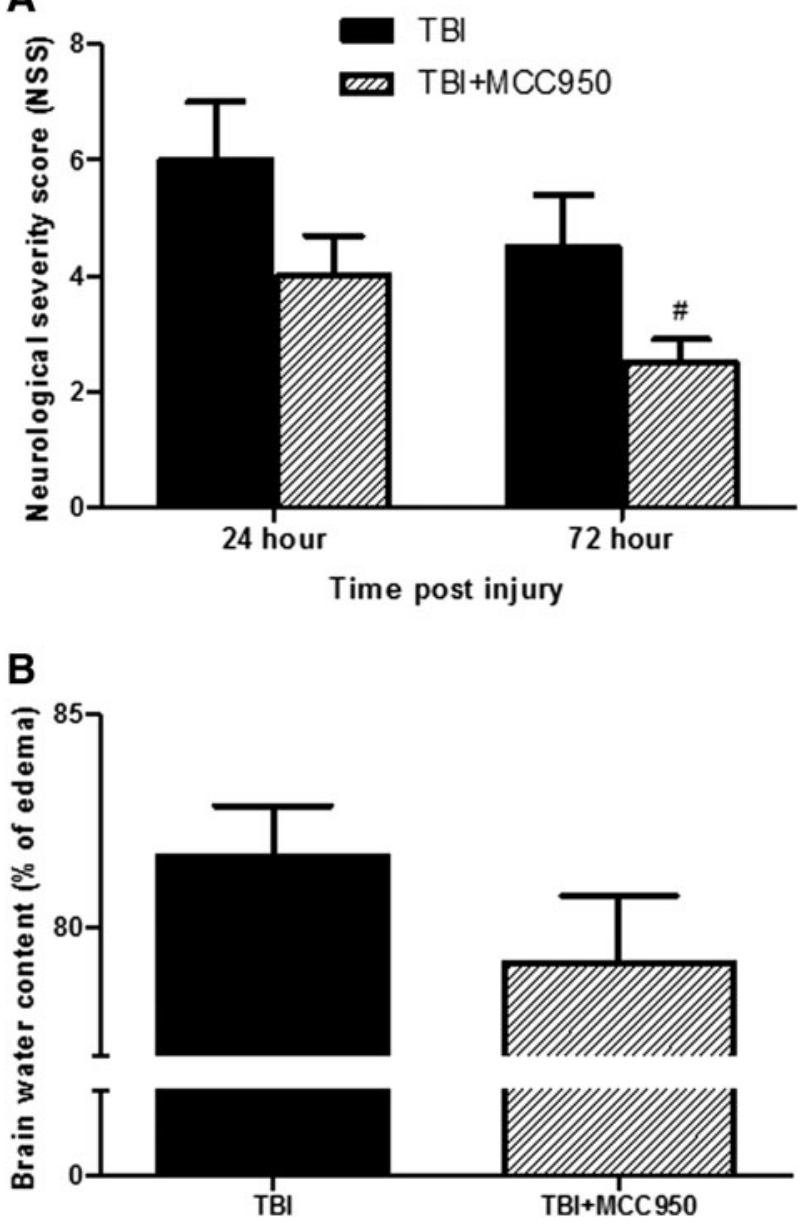

FIG. 1. Effect of MCC950 on neurological severity score and edema post-TBI. (A) C57B1/6 mice were subjected to a 10-point neurobehavioral assessment in 24 and $72 \mathrm{~h}$ post-TBI. Animals treated with MCC950 remarkably showed ameliorated TBI associated neurological impairment. (B) Edema was assessed at $24 \mathrm{~h}$ by measuring brain water content. Administration of MCC950 caused a trend toward the decrease in the edema compared to the saline-treated group, but it was not statistically significant. Values are expressed as mean \pm SEM $(n=12)$. ${ }^{\#} p<0.05$ versus corresponding TBI. SEM, standard error of the mean; TBI, traumatic brain injury.

evidences for TXNIP modulation by Akt signaling, we also estimated Akt activation through the pAkt/Akt ratio (Fig. 5). According to our immunoblot analysis, TBI was associated with a significant $(p<0.05)$ increase in TXNIP expression and Akt activation as determined by pAkt/Akt ratio. MCC950 did not discernibly alter TXNIP level as well as Akt activity at $24 \mathrm{~h}$ post-TBI.

\section{MCC950 treatment represses tumor necrosis factor alpha and nuclear factor kappa B/p65 after traumatic brain injury}

We further elucidated the effect of MCC950 on tumor necrosis factor alpha (TNF- $\alpha$ ) and expression as an immediate pleiotropic response to TBI and NF- $\kappa \mathrm{B} / \mathrm{p} 65$ level (Fig. 6A,B), the transcriptional regulator of inflammatory genes. According to our densitometry analysis, the very low levels of TNF- $\alpha$ expression in sham brains turned to pretty high levels in TBI group $(p<0.05)$, and were 


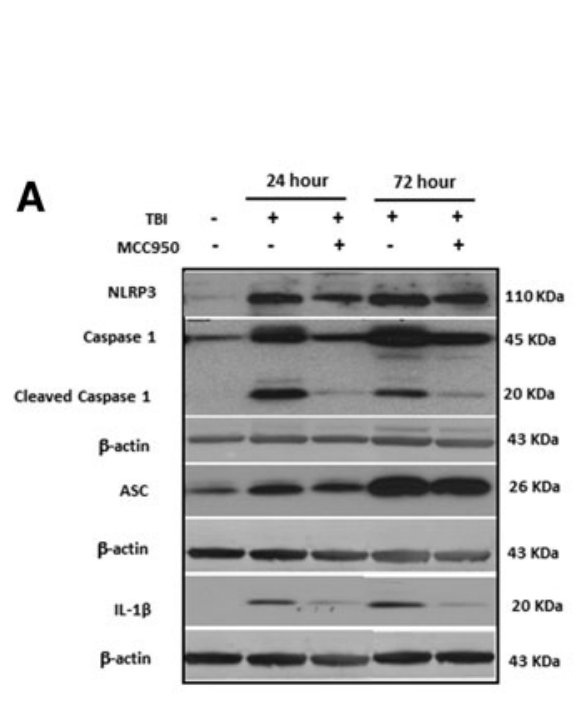

B
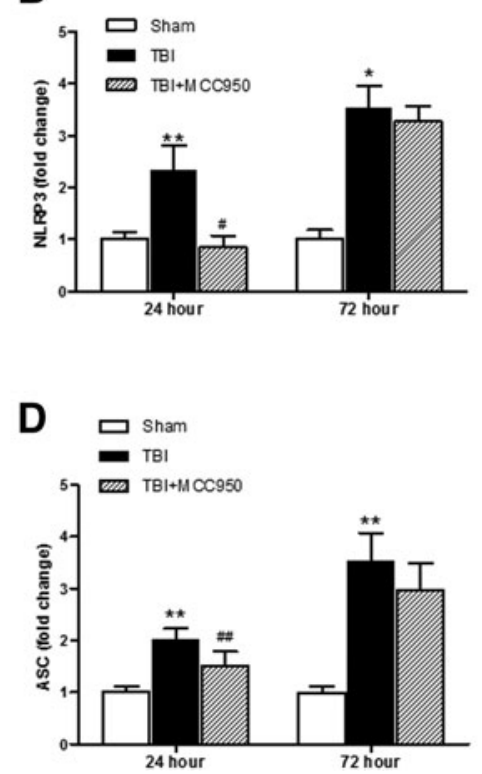
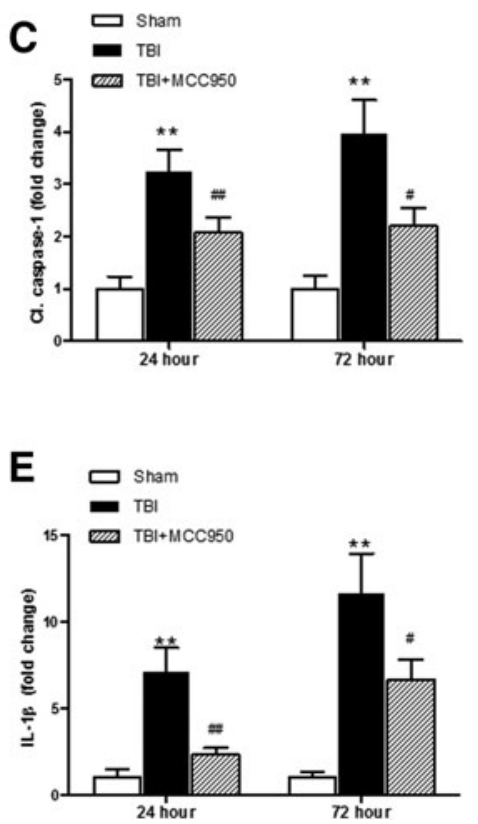

FIG. 2. Effect of MCC950 on expression of principal constituents of NLRP3 inflammasome at 24 and $72 \mathrm{~h}$ post-TBI. (A) Representative and quantitative analysis of (B) NLRP3, (C) cleaved caspase-1, (D) ASC, and (E) IL-1 $\beta$ expression demonstrate the corresponding differences in pericontusional cerebral cortex at 24 and $72 \mathrm{~h}$ post-TBI. MCC 950 treatment post-injury reduced levels of NLRP3 and subsequent caspase- 1 and IL- $1 \beta$ maturation. Values are expressed as mean \pm SEM $(n=8)$. $* p<0.05$ versus corresponding sham; $* * p<0.01$ versus corresponding sham; ${ }^{\#} p<0.05$ versus corresponding TBI; ${ }^{\# \#} p<0.01$ versus corresponding TBI. ASC, apoptosisassociated speck-like; IL-1 $\beta$, interleukin-1 beta; NLRP3, nucleotide oligomerization domain (NOD)-like receptor protein-3; SEM, standard error of the mean; TBI, traumatic brain injury; C1, caspase-1, cleaved caspase-1.

marginally reversed in MCC950-treated TBI animals. Total NF$\kappa \mathrm{B} / \mathrm{p} 65$ also increased in whole-cell lysate in TBI brains. MCC950induced NF- $\kappa \mathrm{B} / \mathrm{p} 65$ repression was rather tangible than TNF- $\alpha$.

\section{MCC950 attenuates activation of caspase-3 and poly (ADP-ribose) polymerase after traumatic brain injury}

To examine the effect of MCC950 on proapoptotic markers, activation of caspase- 3 and PARP was further examined at $24 \mathrm{~h}$ postTBI (Fig. 7A-C). Expression of cleaved PARP and caspase- 3 were significantly increased in TBI mice compared to shams $(p<0.01)$. According to our densitometry analysis in TBI brains, part of this effect is attributed to enhanced levels of the promolecule caspase-3, but less likely to PARP. MCC950-treated brain samples demonstrated a remarkable reduction in PARP and caspase-3 cleavage $(p<0.01)$. The discernible uncleaved caspase- 3 overexpression in TBI animals was also slightly attenuated by MCC950 treatment.

\section{Discussion}

The sufonylurea derivative, MCC950, is among the recently described small-molecule NLRP3 inhibitors developed in an effort to produce a safe, specific leading compound. ${ }^{26}$ It has been considered in several studies for searching for potential therapeutic advantages. In different disease models, MCC950 has provided conspicuous protection either in CNS disease models (i.e., Alzheimer's disease $[\mathrm{AD}]^{27}$ or systemic disorders like diabetic vascular dysfunction) ${ }^{28-31}$ Here, we specified that MCC950 could also alleviate TBI and the consequent proinflammatory and, in particular, proapoptotic signals in the perilesional area.

There is considerable solid evidence highlighting the role of NLRP3 inflammasome in the pathology of CNS disorders that commonly involve neuroinflammation. In this connection, NLRP3 ablation has been shown to ameliorate experimental AD, stroke, Huntington's disease, and pneumococcal meningitis. ${ }^{32-34}$ Our histological evaluations affirm TBI instigated activation of NLRP3 inflammasome in the perilesional area as evident by the increased protein expression of NLRP3, ASC, cleaved caspase-1, and IL-1 $\beta$. Of note, a number of neuroprotective chemicals (e.g., propofol) and natural compounds (e.g., mangniferin) have been shown to provide remarkable NLRP3 repression, which is claimed to explain their therapeutic advantages in TBI. ${ }^{16,18,19,35}$ To our knowledge, MCC950 is the most specifically developed pharmacological tool to accurately address such a contribution. The neurological evaluation of MCC950-treated animals showed a promising improvement. These alleviating effects turned to significant values after $72 \mathrm{~h}$, probably when the effects of molecular events come to affect CNS function. Edema, leading to an increase of brain-water content, has a crucial impact on morbidity and mortality post-TBI because it increases intracranial pressure and contributes to additional secondary injuries. We found that treatment with MCC950 results in a trend toward a decrease in edema in terms of BWC, as expected, but it did not achieve statistical significance. Further, we demonstrated that TBI induces NLRP3 inflammasome activation, as evident by increased protein expression of NLRP3, ASC, caspase-1, cleaved caspase-1, and mature IL-1 $\beta$. Inhibition of NLRP3 inflammasome with the specific small-molecule inhibitor, MCC950, attenuated the corresponding aggravating upregulations in TBI animals. As noted with either immunoblots or immunostained sections, MCC950 treatment demonstrated a differential impact in acute and subacute phases post-TBI. That is, MCC950 repressed all the mentioned inflammasome markers in $24 \mathrm{~h}$ post-injury, with exceptional effects on caspase- 1 and IL-1 $\beta$ maturation persisting to $72 \mathrm{~h}$ post-TBI. Conspicuously, this persisting attenuation of caspase- 1 activation and subsequent IL-1 $\beta$ 


\section{A}

A 24 hour
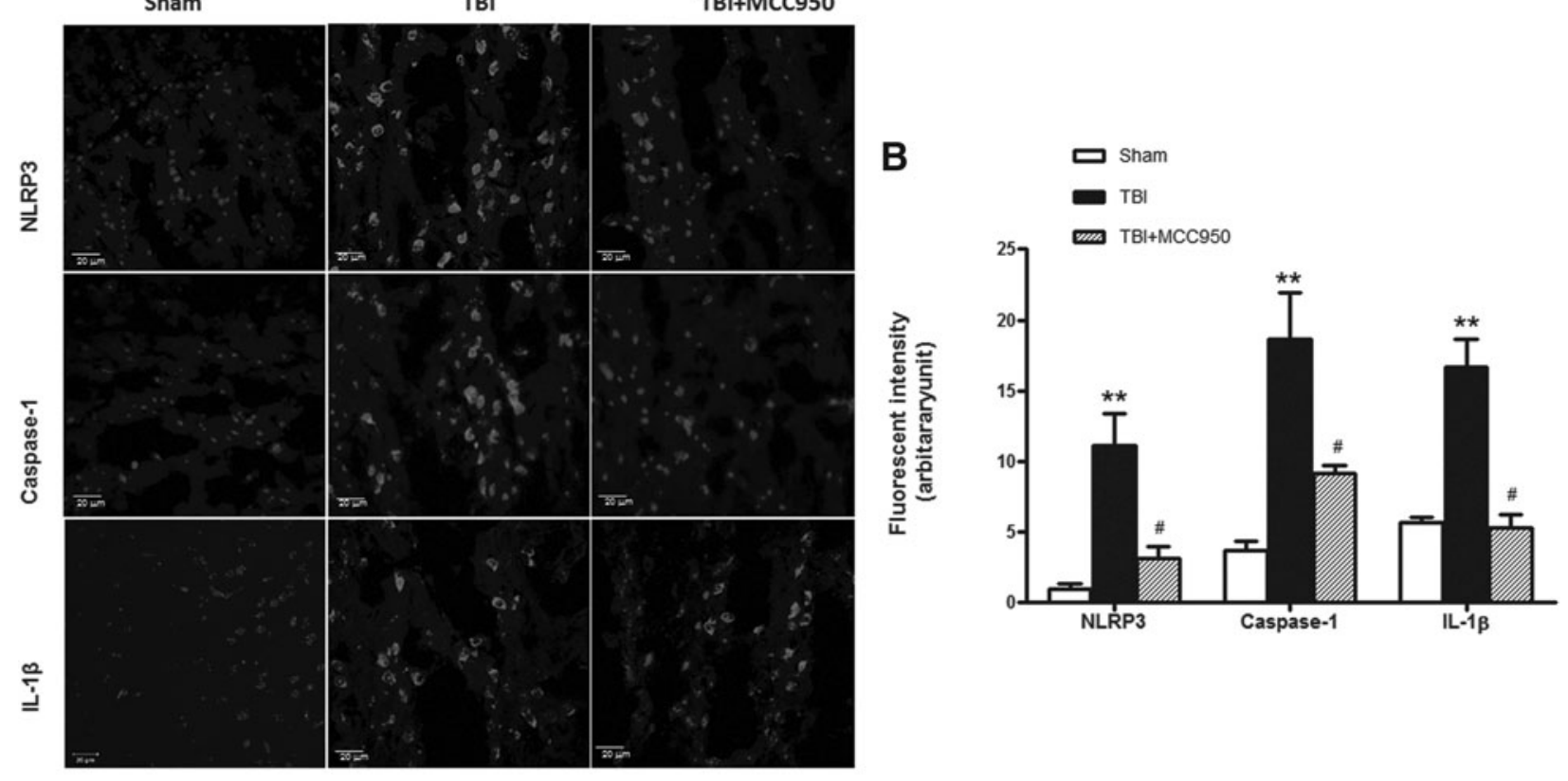

C

72 hour
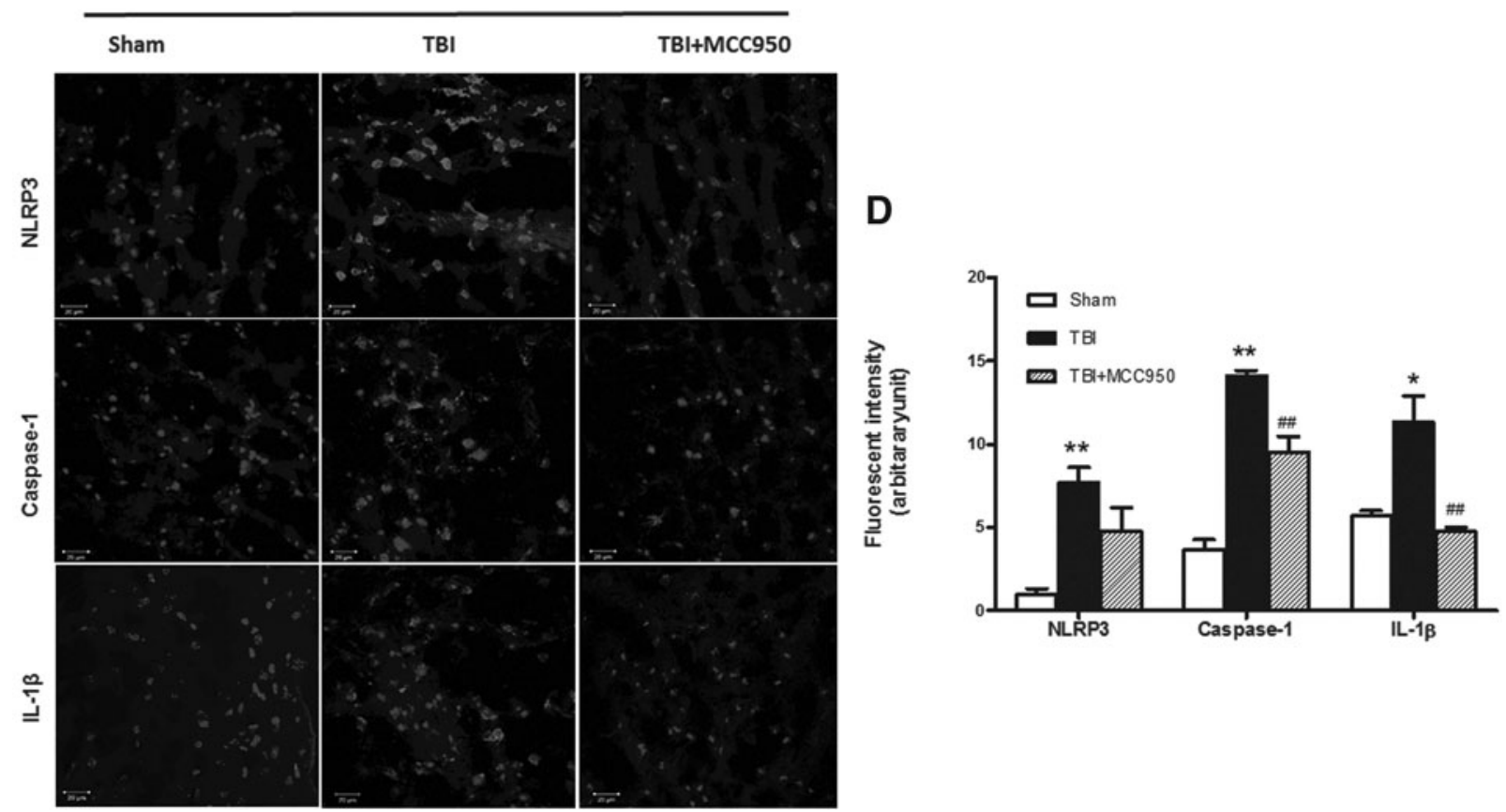

FIG. 3. Effect of MCC 950 on immunohistochemical analysis of principal markers of NLRP3 inflammasome activation at 24 and $72 \mathrm{~h}$ post-TBI. Expression of NLRP3, caspase-1, and IL-1 $\beta$ were evaluated in pericontusional cerebral cortex at 24 (A) and $72 \mathrm{~h}(\mathbf{C})$ post-TBI as an index for acute NLRP3 activation. Fluorescent intensity was calculated and is represented by bar graph (B) and (D). According to the representative probed sections mice treated with MCC950 remarkably reduce TBI-associated NLRP3 inflammasome stimulation $(n=8$; scale bar $=20 \mu \mathrm{m})$. Values are expressed as mean \pm SEM. $* p<0.05$ versus corresponding sham; $* * p<0.01$ versus corresponding sham; ${ }^{\#} p<0.05$ versus corresponding TBI; ${ }^{\# \#} p<0.01$ versus corresponding TBI. CNS, central nervous system; IL- $1 \beta$, interleukin-1 beta; NLRP3, nucleotide oligomerization domain (NOD)-like receptor protein-3; SEM, standard error of the mean; TBI, traumatic brain injury. 

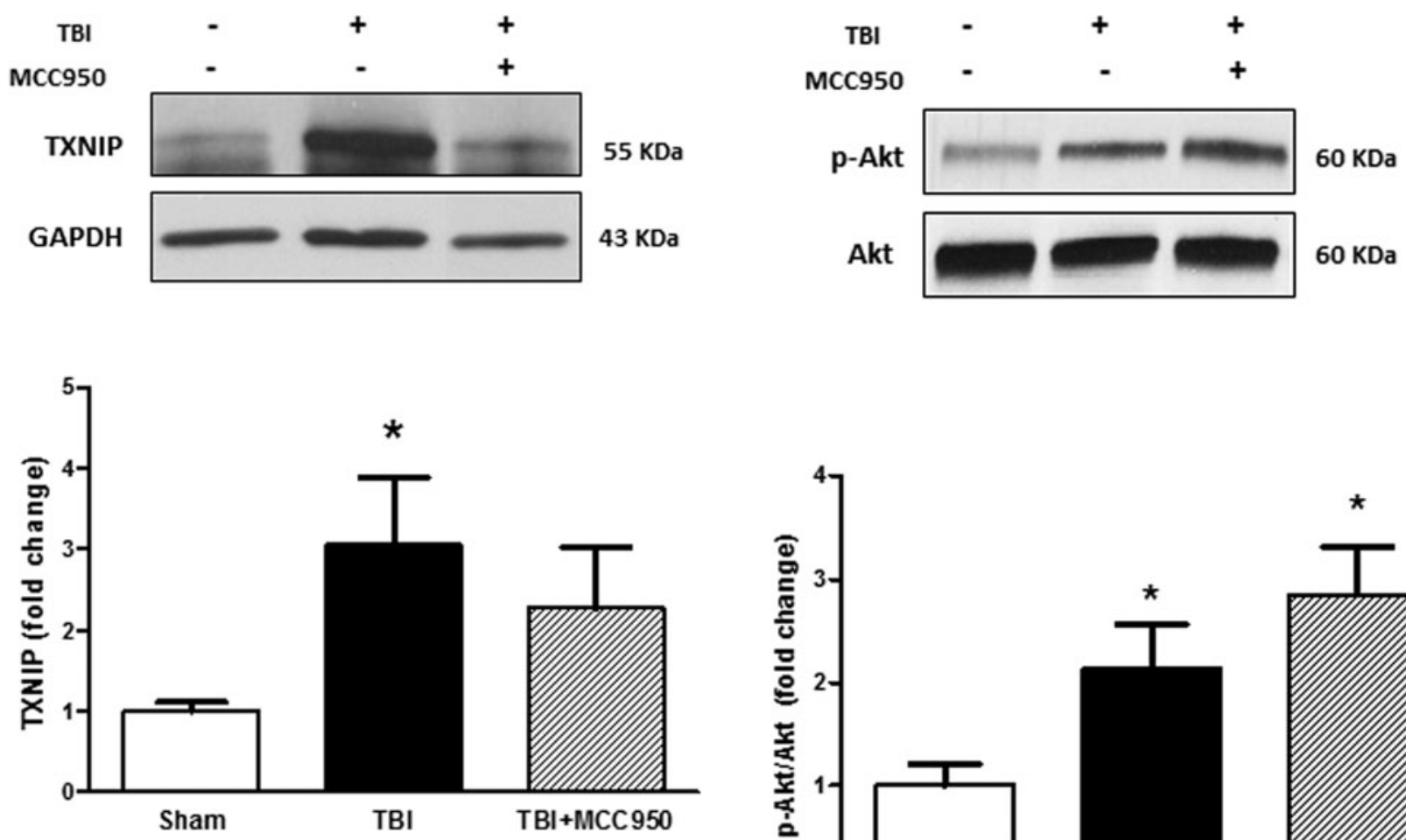

FIG. 4. Effect of MCC950 on TXNIP expression at $24 \mathrm{~h}$ postTBI. Representative and quantitative analysis of western blots showed that TXNIP expression increased significantly at $24 \mathrm{~h}$ in saline-treated TBI mice compared to shams. MCC950 treatment at post-injury induced a minimal decrease in TXNIP levels. Values are expressed as mean $\pm \operatorname{SEM}(n=8) .{ }^{*} p<0.05$ versus sham. GAPDH, glyceraldehyde 3-phosphate dehydrogenase; SEM, standard error of the mean; TBI, traumatic brain injury; TXNIP, thioredoxin interacting protein.

repression parallels with the significant NSS improvement in MCC950-treated TBI animals, a noteworthy observation that may underline the central place of IL- $1 \beta$ in TBI pathology and MCC950 neuroprotection. As a proinflammatory cytokine, IL- $1 \beta$ plays a pivotal role in acute neuronal injury and its blockage has been associated with better prognosis in various clinical conditions. ${ }^{36}$ Clinical studies supports the existence of correlation between IL-1 $\beta$ level and extent of tissue damage post-TBI. ${ }^{37}$

The effect of MCC950 on in TBI-associated inflammation was further evaluated through estimating TNF- $\alpha$ and NF- $\kappa \mathrm{B} / \mathrm{p} 65$ expression. TBI induced a marked expression of TNF- $\alpha$ and the NF$\kappa \mathrm{B} / \mathrm{p} 65$ subunit in the perilesional area. MCC950 treatment was partially efficient in preventing NF- $\kappa \mathrm{B} / \mathrm{p} 65$ upregulation in TBI animals. Even minimal changes in NF- $\kappa \mathrm{B} / \mathrm{p} 65$ might be meaningfully valued in two views. First, it has been shown to be involved in the pathogenesis of TBI. ${ }^{38,39}$ Second, NF- $\kappa \mathrm{B} / \mathrm{p} 65$ is a wellestablished priming signal for NLRP3 inflammasomes. ${ }^{40}$ Transcriptional priming of inflammasome constituents like caspase- 1 is an essential prerequisite step for further NLRP3 inflammasome activation and subsequent IL- $1 \beta$ maturation. ${ }^{41}$ Therefore, the associated NF- $\kappa \mathrm{B} / \mathrm{p} 65$ alterations may provide mechanistic views on the MCC950 mode of action in TBI animals. Based on immunoblots analysis at $24 \mathrm{~h}$, TBI induced a remarkable overexpression of caspase-1 (Supplementary Fig. 1) (see online supplementary material at http://www.liebertpub.com) and caspase-3 as a consequent of the priming signal mediated by NF- $\kappa \mathrm{B} / \mathrm{p} 65$. Interestingly, MCC950-associated NF- $\kappa \mathrm{B} / \mathrm{p} 65$ repression was associated with

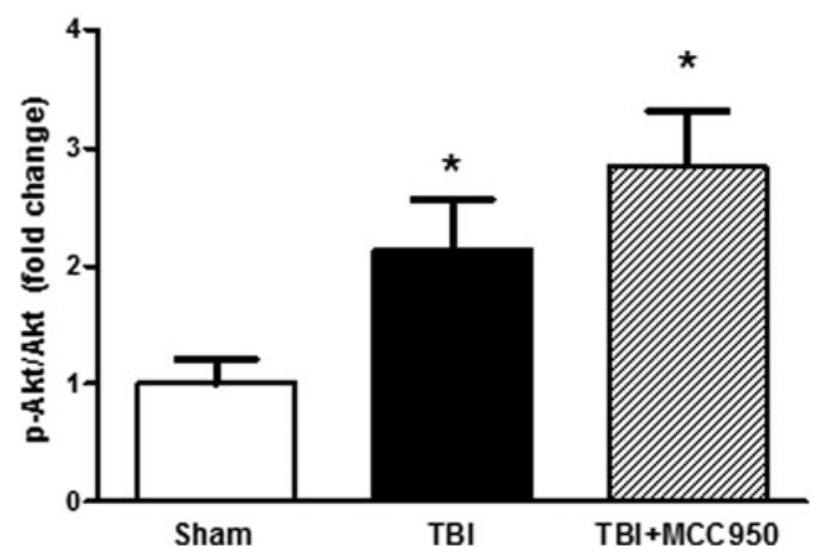

FIG. 5. Effect of MCC 950 on Akt phosphorylation at $24 \mathrm{~h}$ postTBI. Representative and quantitative analysis showed significant elevation in Akt activity as determined by pAkt/Akt ratio in TBI saline-treated and MCC950-treated animals compared to shams. Akt activation was not significantly increased with MCC950 treatment compared to saline TBI only. Values are expressed as mean \pm SEM $(n=8) . * p<0.05$ versus sham. Akt, protein kinase B; pAkt, phosphorylated Akt; SEM, standard error of the mean; TBI, traumatic brain injury.

reduced uncleaved forms of caspase- 1 and caspase-3, indicating that MCC950 is also efficient at blocking the priming step besides its well-characterized inhibitory effect on NLRP3 inflammasome activation and caspase-1 cleavage.

Programmed cell death, namely pyroptosis and apoptosis, is a critical target in TBI given that it leads to irreversible ultimate injury in the salvageable part of the TBI brain. ${ }^{42}$ Pyroptosis could be recognized from apoptosis given that it is triggered by caspase- $1^{43}$ and is associated with the release of proinflammatory cytokines IL- $1 \beta$ and IL-18. ${ }^{44}$ Typically, NLRP3 colocalization with caspease- 1 is an indicator for pyroptosis. Accordingly, the alleviating effects MCC950 in our TBI animals might be somehow linked to attenuated pyroptosis.

Another factor intimately contributing to the pathology of TBI is the apoptosis of neurons and glia. Our TBI brains showed increased protein expression of the proapoptotic molecule, caspase-3, but not that of PARP, at $24 \mathrm{~h}$ post-TBI. Alternatively, the cleavage activation of both molecules was augmented in TBI brains and was strongly prevented by MCC950 treatment, which is highly suggestive of protective effects against apoptotic cell death in the TBI perilesional area.

To provide more details about NLPR3 regulation along with MCC950 treatment, we were also interested to test the corresponding changes in TXNIP and Akt activation. Extensively studied for its contribution to insulin resistance and oxidative stress, TXNIP 
A
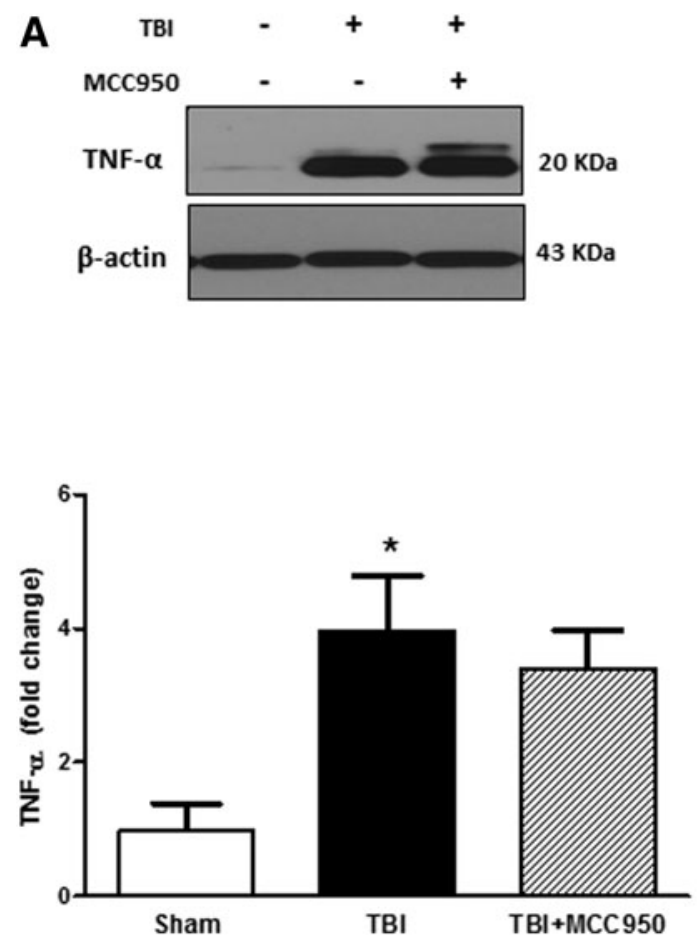
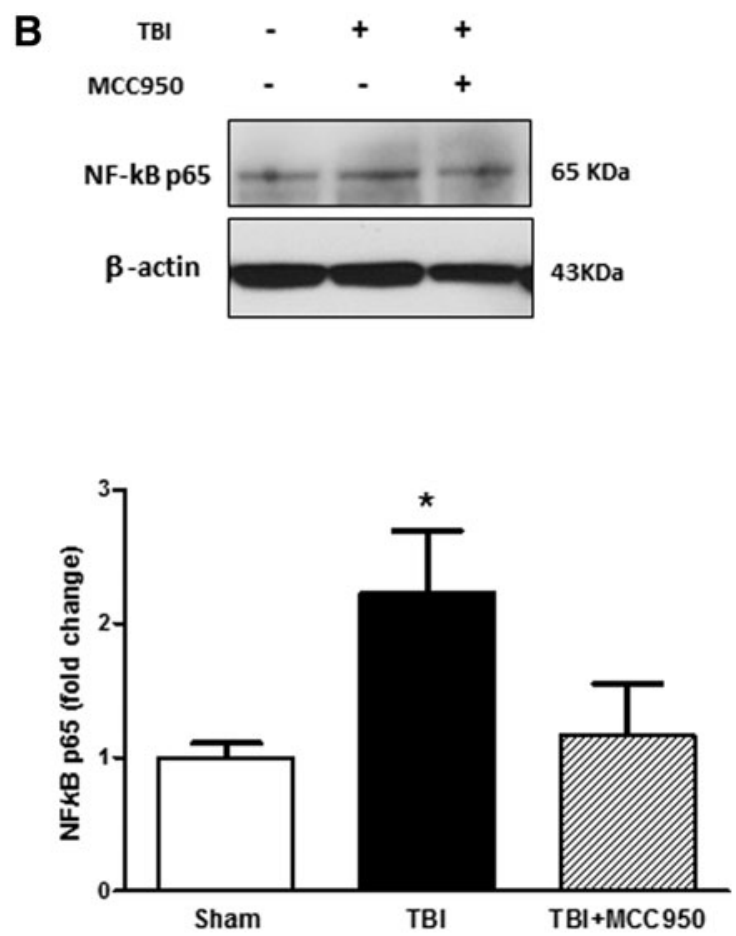

FIG. 6. Effect of MCC 950 on inflammation (TNF- $\alpha$ and NF- $\kappa \mathrm{B} / \mathrm{p} 65)$ at $24 \mathrm{~h}$ post-TBI. Representative and quantitative analysis of western blots showed significant increase in TNF- $\alpha$ (A) and NF- $\kappa \mathrm{B} / \mathrm{p} 65$ (B) expression at $24 \mathrm{~h}$ post-TBI compared to shams. MCC950 treatment showed a trend toward a decrease in NF- $\kappa \mathrm{B} / \mathrm{p} 65$ level. Values are expressed as mean $\pm \mathrm{SEM}(n=8)$. ${ }^{*} p<0.05$ versus sham. $\mathrm{NF}-\kappa \mathrm{B}$, nuclear factor kappa B; SEM, standard error of the mean; TBI, traumatic brain injury; TNF- $\alpha$, tumor necrosis factor alpha.
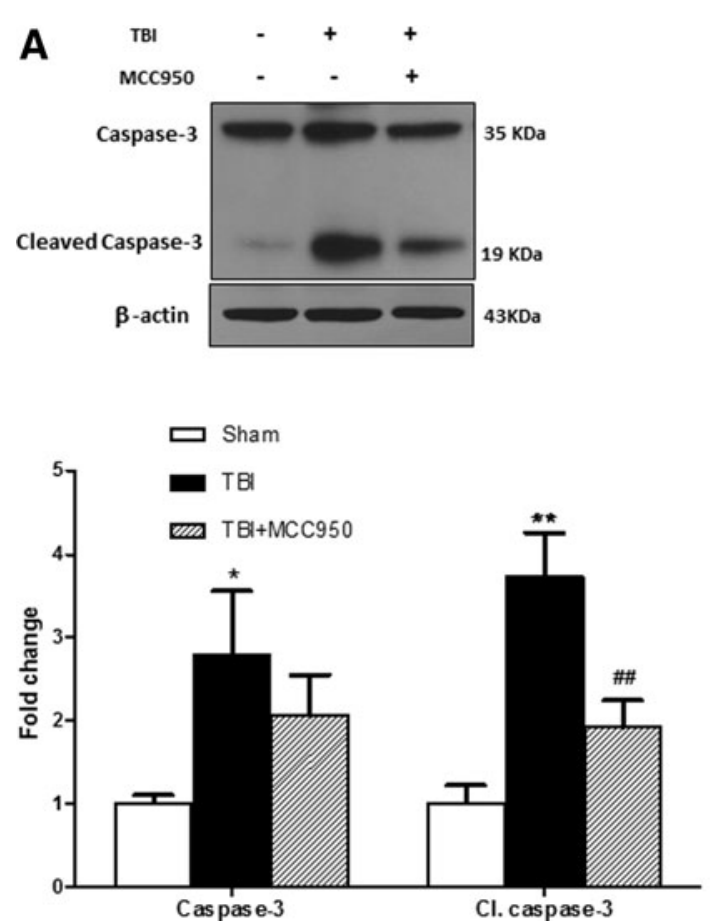
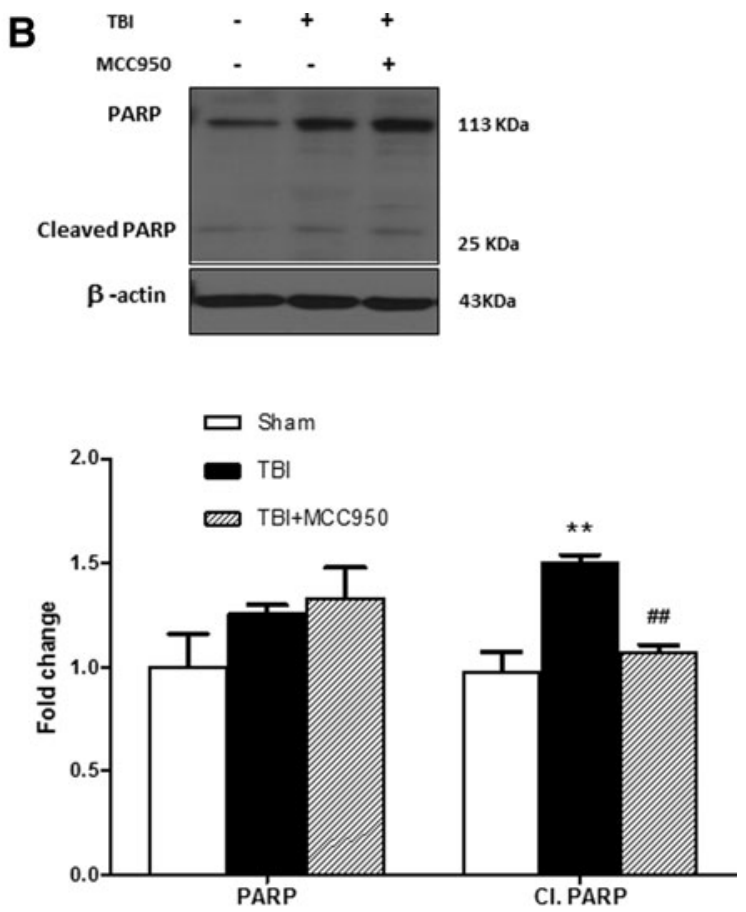

FIG. 7. Effect of MCC950 on expression of proapoptotic markers at $24 \mathrm{~h}$ post-TBI. Representative and quantitative analysis of (A) caspase-3 and cleaved caspse-3 as well as (B) PARP and cleaved PARP immunoblots illustrate significant activation at $24 \mathrm{~h}$ post-TBI. MCC950 treatment significantly attenuated cleavage of both proapoptotic markers. Values are expressed as mean \pm SEM $(n=8)$. ${ }^{*} p<0.05$ versus sham; ${ }^{* *} p<0.01$ versus sham; ${ }^{\# \#} p<0.01$ vs TBI. PARP, poly (ADP-ribose) polymerase; SEM, standard error of the mean; TBI, traumatic brain injury. 


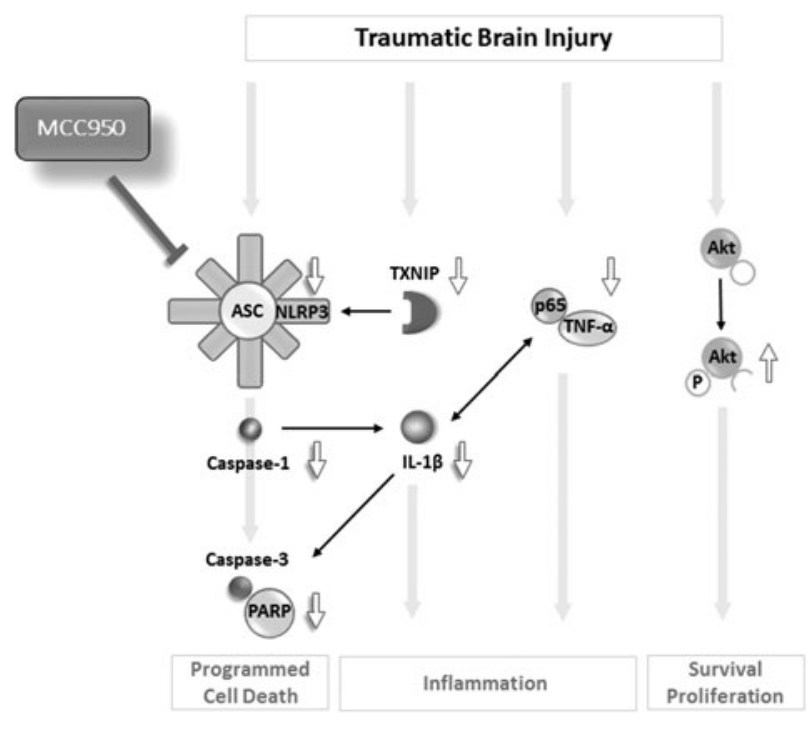

FIG. 8. Pictorial description of MCC950 therapeutic effects on experimental TBI. In a simplified look, TBI instigates several deteriorating signals, that is, through ROS or damage-associated molecular pattern stimulation interfacing with the protective effectors like Akt phosphorylation. The damage signals are sensed by NLRP3 to recruit ASC to NRPL3 inflammasome assembly. TXNIP interacts with NLRP3 inflammasome to augment its cleavage activity on caspase- 1 precursor leading to caspase- 1 and subsequent IL- $1 \beta$ maturation. IL- $1 \beta$ may trigger a wide range of inflammatory reactions, which parallels NF- $\kappa$ B transcriptional activity and TNF- $\alpha$ overproduction. Apoptotic cell death involving caspase- 3 and PARP follows the inflammatory propagation. According to our findings, MCC950 mitigates TBI-induced neuroinflammation and associated apoptotic cascades as determined by arrows with significant changes detected for the shaded ones. Such an ameliorating effect of MCC950 is mainly ascribed to specific inhibition of NLRP3 oligomerization and subsequent IL- $1 \beta$ maturation. Through a likely feedback mechanism, MCC950 may rescue Akt survival activity from deteriorating TBI outcomes. Akt, protein kinase B; pAkt, phosphorylated Akt; ASC, apoptosis-associated speck-like; DAMP, damage-associated molecular pattern; NF- $\kappa \mathrm{B}$, nuclear factor kappa $\mathrm{B}$; NLRP3, nucleotide oligomerization domain (NOD)-like receptor protein-3; PARP, poly (ADP-ribose) polymerase; TBI, traumatic brain injury; ROS, reactive oxygen species; TXNIP, thioredoxin interacting protein; IL- $1 \beta$, interleukin 1 beta; TNF- $\alpha$, tumor necrosis factor alpha; $\mathrm{p} 65, \mathrm{NF}-\kappa \mathrm{B} / \mathrm{p} 65$ subunit.

directly associates with NLRP3 and induces inflammasome oligomerization and activation and modulates the expression of various cytokines and proapoptotic signals. ${ }^{25,29,45,46}$ TXNIP has been also found be upregulated in response to brain injury coincident with inflammasome activation. ${ }^{47}$ Presumptively, this takes place downstream to reactive oxygen species (ROS) release, which, in turn, promotes TXNIP expression trough transcription factor forkhead box O3 (FoxO) in TBI brains. ${ }^{48}$ Based on our immunoblot analysis, TBI induced a significant increase in TXNIP protein, which was slightly modulated by MCC950 treatment.

The serine-threonine kinase, Akt, is implicated in neural survival in acute $\mathrm{TBI}^{49,50}$ and may partially demonstrate MCC950 therapeutic effects on TBI animals. ${ }^{51,52}$ However, Akt phosphorylation post-TBI strictly varied in different cellular compartments. Akt, with its very vast effects, may also independently affect TXNIP transcription and thus subsequent NLRP3 activation. In fact, phosphorylation of FoxOs by activated Akt may inhibit the transcriptional functions of
FoxOs and thus interrupt ROS-induced TXNIP expression. ${ }^{53}$ However, our experiments may not specify such a role for Akt; further investigations are needed to clarify probable involvement of TXNIP and the presumptive regulatory effect of Akt signaling.

This "proof-of-concept study" aims to obtain pre-clinical information to address whether acute treatment with MCC950, a specific NLRP3 inflammasome inhibitor, would improve TBI outcomes. This is the first report indicating that acute MCC950 dosing confers beneficial effects in a mouse model of TBI. However, longer-term studies with multiple days/doses will be needed to assess the actual impact of the manipulation of this promising small-molecule NLRP3 inhibitor. One might expect the impact to be even greater at later time points, where inflammation and apoptosis are even more important contributors to the ultimate secondary damage post-TBI. Further, although unilateral cortical impact injury is a widely used pre-clinical model, given the prevalence of particular TBI forms like the combat blast injury model, it may improve the translational value of preclinical studies on MCC950.

Inflammation is a pivotal degenerating signal persisting in white matter for years in patients with TBI. ${ }^{54}$ Despite several evidences supporting detrimental impact neuroinflammation and edema in TBI patients, no anti-inflammatory drug has been yet been admitted to clinical practice. Even corticosteroids, widely used as unique pluripotent immune-suppressive agents in TBI patients, ${ }^{55}$ have failed to prove beneficiary rather than harmful effects in large clinical trials. ${ }^{56,57}$ Prevalence of infections is another concern in the chronic administration of an immunosuppressive in TBI patients. ${ }^{58}$ As such, specific targeting of major proinflammatory signals may provide efficient and safe medications.

Laboratory studies implicate IL- $1 \beta$ in the pathophysiology of TBI, ${ }^{11}$ supporting investigations on NLRP3 inflammasome inhibitors in pre-clinical studies. The present study provides evidences implicative of a particular place of NLRP3 inflammasome in TBI and the therapeutic potential of MCC950 (Fig. 8). After further examinations, MCC950, as a selective potent NLRP3 inhibitor, might represent a promising candidate for clinical trials for its suitable bioavailability and pharmacokinetic properties. ${ }^{26}$

\section{Acknowledgments}

This work was supported by the National Institutes of Health (R01-NS097800; to T.I.). The authors are thankful to Dr. Anton J. Reiner, for providing a CCI device (Impact One; Leica Biosystems, Buffalo Grove, IL) to induce TBI in mice.

\section{Author Disclosure Statement}

No competing financial interests exist.

\section{References}

1. Majdan, M., Plancikova, D., Maas, A., Polinder, S., Feigin, V., and Theadom, A. (2017). Years of life lost due to traumatic brain injury in Europe: a cross-sectional analysis of 16 countries. PLoS Med. 14, e1002331.

2. Korley, F.K., Kelen, G.D., Jones, C.M., and Diaz-Arrastia, R. (2016). Emergency department evaluation of traumatic brain injury in the United States, 2009-2010. The J. Head Trauma Rehabil. 31, 379-387.

3. Schumacher, M., Denier, C., Oudinet, J.-P., Adams, D., and Guennoun, R. (2016). Progesterone neuroprotection: the background of clinical trial failure. J. Steroid Biochem. Mol. Biol. 160, 53-66.

4. Robertson, C.S., Hannay, H.J., Yamal, J.-M., Gopinath, S., Goodman, J.C., Tilley, B.C., Baldwin, A., Lara, L.R., Saucedo-Crespo, H., and Ahmed, O. (2014). Effect of erythropoietin and transfusion threshold on neurological recovery after traumatic brain injury: a randomized clinical trial. JAMA 312, 36-47. 
5. Corps, K.N., Roth, T.L., and McGavern, D.B. (2015). Inflammation and neuroprotection in traumatic brain injury. JAMA Neurol. 72, 355-362.

6. Pearn, M.L., Niesman, I.R., Egawa, J., Sawada, A., Almenar-Queralt, A., Shah, S.B., Duckworth, J.L., and Head, B.P. (2017). Pathophysiology associated with traumatic brain injury: current treatments and potential novel therapeutics. Cell. Mol. Neurobiol. 37, 571-585.

7. Winkler, E.A., Minter, D., Yue, J.K., and Manley, G.T. (2016). Cerebral edema in traumatic brain injury: pathophysiology and prospective therapeutic targets. Neurosurg. Clin. N. Am. 27, 473-488.

8. Hang, C.-H., Chen, G., Shi, J.-X., Zhang, X., and Li, J.-S. (2006) Cortical expression of nuclear factor $\kappa \mathrm{B}$ after human brain contusion. Brain Res. 1109, 14-21.

9. Zhao, J.-B., Zhang, Y., Li, G.-Z., Su, X.-F., and Hang, C.-H. (2011). Activation of JAK2/STAT pathway in cerebral cortex after experimental traumatic brain injury of rats. Neurosci. Lett. 498, 147-152.

10. Kinoshita, K., Chatzipanteli, K., Vitarbo, E., Truettner, J.S., Alonso, O.F., and Dietrich, W.D. (2002). Interleukin- $1 \beta$ messenger ribonucleic acid and protein levels after fluid-percussion brain injury in rats: importance of injury severity and brain temperature. Neurosurgery 51 195-203.

11. Hutchinson, P.J., O’Connell, M.T., Rothwell, N.J., Hopkins, S.J., Nortje, J., Carpenter, K.L., Timofeev, I., Al-Rawi, P.G., Menon, D.K., and Pickard, J.D. (2007). Inflammation in human brain injury: Intracerebral concentrations of IL- $1 \alpha$, IL-1 $\beta$, and their endogenous inhibitor IL-1ra. J. Neurotrauma. 24, 1545-1557.

12. Henao-Mejia, J., Elinav, E., Strowig, T., and Flavell, R.A. (2012). Inflammasomes: far beyond inflammation. Nat. Immunol. 13, 321-324.

13. Karmakar, M., Katsnelson, M., Malak, H.A., Greene, N.G., Howell, S.J., Hise, A.G., Camilli, A., Kadioglu, A., Dubyak, G.R., and Pearlman, E. (2015). Neutrophil IL- $1 \beta$ processing induced by pneumolysin is mediated by the NLRP3/ASC inflammasome and caspase- 1 activation and is dependent on K+ efflux. J. Immunol. 2015;194:1763-1775.

14. Mohamed, I.N., Ishrat, T., Fagan, S.C., and El-Remessy, A.B. (2015). Role of inflammasome activation in the pathophysiology of vascular diseases of the neurovascular unit. Antioxid. Redox Signal. 22, 11881206.

15. Liu, H.-D., Li, W., Chen, Z.-R., Hu, Y.-C., Zhang, D.-D., Shen, W., Zhou, M.-L., Zhu, L., and Hang, C.-H. (2013). Expression of the NLRP3 inflammasome in cerebral cortex after traumatic brain injury in a rat model. Neurochem. Res. 38, 2072-2083.

16. Fan, K., Ma, J., Xiao, W., Chen, J., Wu, J., Ren, J., Hou, J., Hu, Y. $\mathrm{Gu}$, J., and Yu, B. (2017). Mangiferin attenuates blast-induced traumatic brain injury via inhibiting NLRP3 inflammasome. Chem. Biol. Interact. 271, 15-23

17. Wallisch, J.S., Simon, D.W., Bayir, H., Bell, M.J., Kochanek, P.M., and Clark, R.S.B. (2017). Cerebrospinal fluid NLRP3 is increased after severe traumatic brain injury in infants and children. Neurocrit. Care 27, 44-50.

18. Wei, X., Hu, C.-C., Zhang, Y.-L., Yao, S.-L., and Mao, W.-K. (2016). Telmisartan reduced cerebral edema by inhibiting NLRP3 inflammasome in mice with cold brain injury. J. Huazhong Univ. Sci. Technolog. Med. Sci. 36, 576-583.

19. Lin, C., Chao, H., Li, Z., Xu, X., Liu, Y., Bao, Z., Hou, L., Liu, Y., Wang, X., and You, Y. (2017). Omega-3 fatty acids regulate NLRP3 inflammasome activation and prevent behavior deficits after traumatic brain injury. Exp. Neurol. 290, 115-122.

20. Kurokawa, H., Sugiyama, S., Nozaki, T., Sugamura, K., Toyama, K., Matsubara, J., Fujisue, K., Ohba, K., Maeda, H., and Konishi, M. (2015). Telmisartan enhances mitochondrial activity and alters cellular functions in human coronary artery endothelial cells via AMPactivated protein kinase pathway. Atherosclerosis 239, 375-385.

21. Malik, S., Suchal, K., Gamad, N., Dinda, A.K., Arya, D.S., and Bhatia, J. (2015). Telmisartan ameliorates cisplatin-induced nephrotoxicity by inhibiting MAPK mediated inflammation and apoptosis. Eur. J. Pharmacol. 748, 54-60.

22. Coll, R.C., Robertson, A.A., Chae, J.J., Higgins, S.C., Muñoz-Planillo, R., Inserra, M.C., Vetter, I., Dungan, L.S., Monks, B.G., and Stutz, A. (2015). A small-molecule inhibitor of the NLRP3 inflammasome for the treatment of inflammatory diseases. Nat. Med. 21, 248-255.

23. Primiano, M.J., Lefker, B.A., Bowman, M.R., Bree, A.G., Hubeau, C., Bonin, P.D., Mangan, M., Dower, K., Monks, B.G., and Cushing, L. (2016). Efficacy and pharmacology of the NLRP3 inflammasome inhibitor CP-456,773 (CRID3) in murine models of dermal and pulmonary inflammation. J. Immunol. 197, 2421-2433.
24. van Hout, G.P., Bosch, L., Ellenbroek, G.H., de Haan, J.J., van Solinge, W.W., Cooper, M.A., Arslan, F., de Jager, S.C., Robertson, A.A., and Pasterkamp, G. (2016). The selective NLRP3-inflammasome inhibitor MCC950 reduces infarct size and preserves cardiac function in a pig model of myocardial infarction. Eur. Heart J. 38, 828-836.

25. Ishrat, T., Mohamed, I.N., Pillai, B., Soliman, S., Fouda, A.Y., Ergul, A., El-Remessy, A.B., and Fagan, S.C. (2015). Thioredoxininteracting protein: a novel target for neuroprotection in experimental thromboembolic stroke in mice. Mol. Neurobiol. 51, 766-778.

26. Salla, M., Butler, M.S., Pelingon, R., Kaeslin, G., Croker, D.E., Reid, J.C., Baek, J.M., Bernhardt, P.V., Gillam, E.M., and Cooper, M.A. (2016). Identification, synthesis, and biological evaluation of the major human metabolite of NLRP3 inflammasome inhibitor MCC950. ACS Med. Chem. Lett. 7, 1034-1038.

27. Dempsey, C., Araiz, A.R., Bryson, K., Finucane, O., Larkin, C., Mills, E., Robertson, A., Cooper, M., O’Neill, L., and Lynch, M. (2017). Inhibiting the NLRP3 inflammasome with MCC950 promotes nonphlogistic clearance of amyloid- $\beta$ and cognitive function in APP/PS1 mice. Brain Behav. Immun. 61, 306-316.

28. Dinh, Q.N., Drummond, G.R., Kemp-Harper, B.K., Diep, H., De Silva, T.M., Kim, H.A., Vinh, A., Robertson, A.A., Cooper, M.A., and Mansell, A. (2017). Pressor response to angiotensin II is enhanced in aged mice and associated with inflammation, vasoconstriction and oxidative stress. Aging 9, 1595-1606.

29. Zhao, Q., Che, X., Zhang, H., Fan, P., Tan, G., Liu, L., Jiang, D., Zhao, J., Xiang, X., and Liang, Y. (2017). Thioredoxin-interacting protein links endoplasmic reticulum stress to inflammatory brain injury and apoptosis after subarachnoid haemorrhage. J. Neuroinflammation 14, 104.

30. Wali, B., Stein, D.G., and Sayeed, I. (2017). Intralipid Vehicle Does Not Interfere with the Efficacy of Progesterone in Attenuating Edema following Traumatic Brain Injury. J. Neurotrauma 34, 2183-2186.

31. Mridha, A.R., Wree, A., Robertson, A.A., Yeh, M.M., Johnson, C.D., Van Rooyen, D.M., Haczeyni, F., Teoh, N.C., Savard, C., and Ioannou, G.N. (2017). NLRP3 inflammasome blockade reduces liver inflammation and fibrosis in experimental NASH in mice. J. Hepatol. 66, 1037-1046.

32. Hoegen, T., Tremel, N., Klein, M., Angele, B., Wagner, H., Kirschning, C., Pfister, H.-W., Fontana, A., Hammerschmidt, S., and Koedel, U. (2011). The NLRP3 inflammasome contributes to brain injury in pneumococcal meningitis and is activated through ATP-dependent lysosomal cathepsin B release. J. Immunol. 187, 5440-5451.

33. Halle, A., Hornung, V., Petzold, G.C., Stewart, C.R., Monks, B.G., Reinheckel, T., Fitzgerald, K.A., Latz, E., Moore, K.J., and Golenbock, D.T. (2008). The NALP3 inflammasome is involved in the innate immune response to amyloid- $\beta$. Nat. Immunol. 9, 857-865.

34. Yang, F., Wang, Z., Wei, X., Han, H., Meng, X., Zhang, Y., Shi, W., Li, F., Xin, T., and Pang, Q. (2014). NLRP3 deficiency ameliorates neurovascular damage in experimental ischemic stroke. J. Cereb. Blood Flow Metab. 34, 660-667.

35. Ma, J., Xiao, W., Wang, J., Wu, J., Ren, J., Hou, J., Gu, J., Fan, K., and $\mathrm{Yu}, \mathrm{B}$. (2016). Propofol inhibits NLRP3 inflammasome and attenuates blast-Induced traumatic brain injury in rats. Inflammation 39, 2094-2103.

36. Murray, K.N., Parry-Jones, A.R., and Allan, S.M. (2015). Interleukin1 and acute brain injury. Front. Cell. Neurosci. 9, 18.

37. Tasci, A., Okay, O., Gezici, A.R., Ergun, R., and Ergungor, F. (2003). Prognostic value of interleukin-1 beta levels after acute brain injury. Neurol. Res. 25, 871-4.

38. Nonaka, M., Chen, X.-H., Pierce, J.E., Leoni, M.J., McIntosh, T.K., Wolf, J.A., and Smith, D.H. (1999). Prolonged activation of NF- $\kappa$ B following traumatic brain injury in rats. J. Neurotrauma 16, 1023-1034.

39. Wang, Z.-R., Li, Y.-X., Lei, H.-Y., Yang, D.-Q., Wang, L.-Q., and Luo, M.-Y. (2016). Regulating effect of activated NF- $\kappa$ B on edema induced by traumatic brain injury of rats. Asian Pac. J. Trop. Med. 9, 274-277.

40. Juliana, C., Fernandes-Alnemri, T., Kang, S., Farias, A., Qin, F., and Alnemri, E.S. (2012). Non-transcriptional priming and deubiquitination regulate NLRP3 inflammasome activation. J. Biol. Chem. 287, 36617-36622.

41. Ishrat, T., and Nasoohi, S. (2018). The NLRP3 inflammasome: a possible therapeutic target for treatment of stroke, in: Cellular and Molecular Approaches to Regeneration and Repair. Springer: Cham, Switzerland, pps. 427-480. 
42. Su, N., Fei, F., and Fei, Z. (2016). Mechanisms of programmed cell death and molecular pathways in traumatic brain injury. Available at: http://www.avidscience.com/wp-content/uploads/2017/03/PCD-16-01 .pdf. Accessed February 25, 2018.

43. Miao, E.A., Rajan, J.V., and Aderem, A. (2011). Caspase-1-induced pyroptotic cell death. Immunol. Rev. 243, 206-214.

44. Jorgensen, I., and Miao, E.A. (2015). Pyroptotic cell death defends against intracellular pathogens. Immunol. Rev. 265, 130-142.

45. Perrone, L., Devi, T.S., Hosoya, K.I., Terasaki, T., and Singh, L.P. (2009). Thioredoxin interacting protein (TXNIP) induces inflammation through chromatin modification in retinal capillary endothelial cells under diabetic conditions. J. Cell. Physiol. 221, 262-272.

46. Zhou, R., Yazdi, A.S., Menu, P., and Tschopp, J. (2011). A role for mitochondria in NLRP3 inflammasome activation. Nature 469, 221.

47. Kim, G.S., Jung, J.E., Narasimhan, P., Sakata, H., and Chan, P.H. (2012). Induction of thioredoxin-interacting protein is mediated by oxidative stress, calcium, and glucose after brain injury in mice. Neurobiol. Dis. 46, 440-449.

48. Li, X., Rong, Y., Zhang, M., Wang, X.L., LeMaire, S.A., Coselli, J.S., Zhang, Y., and Shen, Y.H. (2009). Up-regulation of thioredoxin interacting protein (Txnip) by $\mathrm{p} 38$ MAPK and FOXO1 contributes to the impaired thioredoxin activity and increased ROS in glucosetreated endothelial cells. Biochem. Biophys. Res. Commun. 381, 660-665.

49. Farook, J., Shields, J., Tawfik, A., Markand, S., Sen, T., Smith, S.B., Brann, D., Dhandapani, K.M., and Sen, N. (2013). GADD34 induces cell death through inactivation of Akt following traumatic brain injury. Cell Death Dis. 4, e754.

50. Ge, X.-T., Lei, P., Wang, H.-C., Zhang, A.-L., Han, Z.-L., Chen, X., Li, S.-H., Jiang, R.-C., Kang, C.-S., and Zhang, J.-N. (2014). miR-21 improves the neurological outcome after traumatic brain injury in rats. Sci. Rep. 4, 6718

51. Zhao, S., Fu, J., Liu, X., Wang, T., Zhang, J., and Zhao, Y. (2012). Activation of Akt/GSK-3beta/beta-catenin signaling pathway is involved in survival of neurons after traumatic brain injury in rats. Neurol. Res. 34, 400-407.
52. Noshita, N., Lewén, A., Sugawara, T., and Chan, P.H. (2002). Akt phosphorylation and neuronal survival after traumatic brain injury in mice. Neurobiol. Dis. 9, 294-304.

53. Hay, N. (2011). Interplay between FOXO, TOR, and Akt. Biochim. Biophys. Acta 1813, 1965-1970.

54. Johnson, V.E., Stewart, J.E., Begbie, F.D., Trojanowski, J.Q., Smith, D.H., and Stewart, W. (2013). Inflammation and white matter degeneration persist for years after a single traumatic brain injury. Brain 136, 28-42.

55. Alderson, P., and Roberts, I. (2005). Corticosteroids for acute traumatic brain injury. Cochrane Database Syst. Rev. (1), CD000196.

56. Roberts, I., Yates, D., Sandercock, P., Farrell, B., Wasserberg, J., Lomas, G., Cottingham, R., Svoboda, P., Brayley, N., and Mazairac, G. (2004). Effect of intravenous corticosteroids on death within 14 days in 10008 adults with clinically significant head injury (MRC CRASH trial): randomised placebo-controlled trial. Lancet 364, 1321-1328.

57. CRASH Trial Collaborators. (2005). Final results of MRC CRASH, a randomised placebo-controlled trial of intravenous corticosteroid in adults with head injury-outcomes at 6 months. Lancet 365, 1957-1959.

58. Kourbeti, I., Vakis, A., Papadakis, J., Karabetsos, D., Bertsias, G., Filippou, M., Ioannou, A., Neophytou, C., Anastasaki, M., and Samonis, G. (2012). Infections in traumatic brain injury patients. Clin. Microbiol. Infect. 18, 359-364.

Address correspondence to:

Tauheed Ishrat, PhD

Department of Anatomy and Neurobiology University of Tennessee Health Science Center

College of Medicine

855 Monroe Avenue

Wittenborg Building

Room-231

Memphis, TN 38163

E-mail: tishrat@uthsc.edu 